\title{
Postictal clinical and EEG activity following intracranially recorded bilateral tonic-clonic seizures
}

\author{
Lisa M Bateman, MD ${ }^{1}$, Anil Mendiratta, MD'1, Jyun-you Liou, MD², Elliot J Smith, PhD $^{3}$, Carl \\ W Bazil, MD, PhD ${ }^{1}$, Hyunmi Choi, MD ${ }^{1}$, Guy M McKhann, MD ${ }^{3}$, Alison Pack, MD ${ }^{1}$, Shraddha \\ Srinivasan, $\mathbf{M D}^{1}$, and Catherine A Schevon, MD, $\mathbf{P h D}^{1}$ \\ ${ }^{1}$ Department of Neurology, Columbia University Medical Center \\ ${ }^{2}$ Department of Physiology and Cellular Biophysics, Columbia University Medical Center \\ ${ }^{3}$ Department of Neurological Surgery, Columbia University Medical Center
}

\section{Summary}

Objective: The dynamics of the postictal period, which may demonstrate such dramatic clinical phenomena as focal neurological deficits, prolonged coma and immobility, and even sudden death, are poorly understood. We sought to classify and characterize postictal phases of bilateral tonicclonic seizures based on EEG criteria and associated clinical features.

Methods: We performed a detailed electroclinical evaluation of the postictal period in a series of 31 bilateral tonic-clonic seizures in 16 patients undergoing epilepsy surgery evaluations for focal pharmacoresistant epilepsy with intracranial electrodes and time-locked video.

Results: The postictal EEG demonstrated three clearly differentiated phases: attenuation, a burstattenuation pattern, and a return to continuous background, with abrupt, synchronized transitions between phases. Postictal attenuation was common, occurring in $84 \%$ of seizures in $94 \%$ of patients in this study. There was increased power in gamma frequencies $(>25 \mathrm{~Hz})$ during postictal attenuation periods relative to preictal baseline in $88 \%$ of seizures demonstrating the attenuation pattern ( $\mathrm{N}=25$ seizures, $\mathrm{p}<0.002$ ). Such increases were seen in more than $90 \%$ of channels in 13 seizures (52\%), and fewer than $10 \%$ of channels in three seizures (12\%). Postictal immobility was seen in $87 \%$ of seizures, with either a flaccid $(58 \%)$ or rigid/dystonic (29\%) appearance. Clinical motor manifestations, including focal dystonic posturing, automatisms, head and eye deviation, and myoclonic jerking, continued or emerged within the first minute following seizure termination in $48 \%$ of seizures, regardless of EEG appearance.

Significance: Intracranial postictal attenuation, which may be diffuse or focal, is so common that it should be regarded as an ubiquitous feature of bilateral tonic-clonic seizures, rather than an unusual event. The prominence of high frequency activity coupled with emerging clinical features,

Corresponding Author: Lisa M. Bateman, MD, FRCPC, 710 West $168^{\text {th }}$ Street, New York NY 10032, Tel: 212-305-1742, Fax: 212-305-5445, 1b2863@cumc.columbia.edu.

Ethical Publication Statement

We confirm that we have read the Journal's position on issues involved in ethical publication and affirm that this report is consistent with those guidelines. 
including rigid immobility and semiologies such as automatisms, during the postictal period support the presence of ongoing seizure-related neuronal activity in unrecorded brain regions.

\section{Keywords}

EEG; Intracranial EEG; Focal epilepsy; high frequency activity; postictal state

\section{Introduction}

Recently, there has been increasing interest in the dynamics of seizure termination and the postictal period. It has commonly been assumed that seizures and their immediate clinical manifestations stop simultaneously, after which there is a period of gradual postictal recovery. In reality, seizure activity may continue in some regions after having ceased in others ${ }^{1}$, and the postictal period may contain striking electrographic or clinical features. Postictal EEG analysis has largely focused on regional slowing or attenuation ${ }^{2}$ and postictal generalized EEG suppression, or PGES ${ }^{3}$. Despite the attention paid to these interesting features, their cellular origins remain incompletely described. Regional attenuation is thought to represent relative neuronal inactivity, and is considered to have localizing value ${ }^{2}$. Going further, some groups have hypothesized that the extreme state of diffuse EEG attenuation is a marker of "cerebral electrical shutdown", a highly clinically significant event with implications for post-ictal morbidity and mortality ${ }^{3-5}$.

Using an existing dataset of retrospectively acquired intracranial EEG recordings of focal to bilateral tonic-clonic seizures (FBTCS) with time-locked video, we undertook a systematic investigation of the post-seizure period in order to develop hypotheses regarding brain states after the apparent cessation of electrographic seizure activity recorded from the cortex. We defined an intracranial version of EEG attenuation in the frequencies most often visualized in clinical recordings (intracranial postictal attenuation, IPA), and assessed for the copresence of gamma rhythms. These are typically better visualized on intracranial recordings than scalp $\mathrm{EEG}^{6,7}$, and are considered to serve as an index of local neuronal activity. ${ }^{8-10} \mathrm{By}$ combining the different types of EEG data and clinical observations, we propose a multistage structure of the postictal state that is far more complex than a gradual recovery from the point of electrographic seizure termination in cortical recordings, and may indicate ongoing activity in other brain areas.

\section{Methods}

Patients aged 16 - 60 years who were studied with intracranial (subdural and/or depth) electrodes at Columbia University Medical Center/New York Presbyterian Hospital between 2005 and 2015, and had at least one FBTCS recorded with a sampling rate per channel of at $500 \mathrm{~Hz}(0.5-125 \mathrm{~Hz}$ bandpass, XLTek clinical EEG systems, Natus Medical Inc., Pleasanton, CA) were included in this retrospective study. In all cases, the reference was either an epidural electrode facing away from the brain (for subdural recordings) or an epidural bolt (for stereotactic depth recordings). Henceforth, we use the term "seizure" to signify the FBTCS under study. Seizures with insufficient peri-ictal video EEG data or excessive artifact, inadequate visualization of the patient, or fewer than 25 electrodes with 
good quality recording at the time of the seizure were excluded. The study was approved by the Institutional Review Board of Columbia University Medical Center, with a waiver of informed consent due to the study's retrospective nature.

Extended video EEG clips beginning at least five minutes before seizure onset and continuing through one hour after seizure termination were reviewed by two board-certified electroencephalographers (LMB and CAS), using Natus review software with embedded antialiasing filters ( $4^{\text {th }}$ order Butterworth) at a time resolution setting of $30 \mathrm{~mm} / \mathrm{sec}$ and sensitivity of $50 \mu \mathrm{V} / \mathrm{mm}$. In order to distinguish the results of our analysis from similar prior studies in scalp EEG recordings ${ }^{3}$, we introduce the term IPA, defined as signal amplitude of less than $50 \mu \mathrm{V}$ in all recorded channels with no discernable physiological activity in the delta, theta, alpha, and beta frequency bands, apart from movement, line noise, and electrode artifacts (Figure 1A). The threshold of $50 \mu \mathrm{V}$ was selected based on the criteria for PGES in scalp recordings of $10 \mu \mathrm{V}$, and a conservatively assumed amplitude ratio of intracranial to scalp EEG of 5:1. ${ }^{11,12}$ The times of the following transitions were visually identified by consensus of the two reviewers: seizure termination, IPA onset, end of continuous attenuation and transition to a discontinuous background pattern, defined by a stuttering return to continuity, with bursts of activity interspersed with periods of relative attenuation, and onset of continuous background. Seizure termination was defined as the end time of high amplitude, widely distributed epileptic discharges time-locked with clonic movements. In cases with asynchronous seizure offsets, seizure termination was identified as the final offset time.

Continuous time-locked video was reviewed between seizure onset and the resumption of continuous background activity, blinded to the EEG-defined transitions by viewing the video full-screen. Seizures were categorized into subclasses as described in ${ }^{13}$ : bilateral and symmetric tonic arm extension (Type 1), clonic jerking without tonic extension or flexion (Type 2), and asymmetric tonic extension or flexion (Type 3). Postictal immobility was defined as the patient becoming motionless following cessation of clonic activity ${ }^{14}$ and further categorized as flaccid, in which the patient appeared limp with normal or reduced muscle tone, or rigid, characterized by fixed limb posturing, e.g. with arms held out stiffly and remaining in position even after the limb was moved by staff. At times, rigid immobility was accompanied by transient episodes of focal dystonic posturing. Onset and offset times for the following clinical features were determined: immobility, oral or manual automatisms, clonic jerking, bicycling or other focal motor features, dystonic posturing, head and/or eye deviation, and myoclonic jerking. Correlation of onset and offset times for clinical features to EEG transitions was assessed using the Wilcoxon rank-sum test with a significance value of 0.05 . Standard deviations were reported where applicable.

Spectral analysis of EEG was conducted using custom software implemented in Matlab (Mathworks, Natick, MA). Power spectral density was examined using the short-time Fourier transform (Matlab "spectrogram" function), and compared to the five-minute preictal baseline, excluding herald/sentinel spikes. Typically during IPA, power below the gamma band (i.e. below $25 \mathrm{~Hz}$ ) was reduced compared to baseline, while gamma power was either reduced or increased. We classified channels according to whether gamma power was increased during IPA, using a threshold defined as mean power plus five times the 
coefficient of variation computed from the pre-ictal baseline. ${ }^{15}$ To determine whether the number of channels with increased gamma power was statistically greater than zero (the null hypothesis), we used a resampling technique. Epochs were randomly selected from the baseline recording, with epoch duration equal to duration of the IPA period, and the probability of exceeding the aforementioned threshold was determined per channel over 100 trials. One seizure with less than five minutes of pre-ictal baseline recording was excluded from this calculation (Table 1). Statistical significance was assessed using the Wilcoxon sign rank test, with Bonferroni correction for multiple comparisons.

\section{Results}

Sixteen patients (10 men, 6 women) met the study criteria, with demographic information given in Table 1. Mean age was $28.1 \pm 8.7$ years (range 16-50). In total, 31 FBTCS were included (mean 1.9 seizures per patient). Eleven seizures (35\%) were classified as Type 1, four seizures (13\%) as Type 2, and $16(52 \%)$ as Type 3. Cessation of bilateral clonic jerking was noted to precede electrographic termination in eight seizures (26\%).

The number of implanted electrodes ranged from 48 to 128 (mean $89.6 \pm 31.7$ ). Of the sixteen patients, three had bilateral coverage, two with stereotactically placed depth arrays, and one with bilateral strips and depths. Eleven patients had broad unilateral coverage, with four patients sampling locations throughout the hemisphere, two patients sampling three lobes, and five with extensive frontal and temporal coverage. The remaining two patients had implants restricted to the left temporal and left parietal lobes, respectively.

\section{Postictal EEG characteristics}

Seizure termination was simultaneous in 24 seizures (77\%), while in seven seizures (23\%), termination was asynchronous (Figure 1C). Focal runs of ictal discharges (typically limited to 1-2 channels) were noted during the first minute after final seizure termination in eight seizures (26\%, example in Figure 1C). Four seizures were triggered during electrical stimulation mapping (Table 1). All but one of the patients (Patient 12) with provoked seizures had additional spontaneous events recorded that were also part of the study.

The post-ictal EEG recording was characterized by three distinct phases, not all of which occurred after every seizure. The first phase, IPA, was characterized by marked attenuation $(<50 \mu \mathrm{V})$ with no discernable physiological rhythms below the gamma range. Examples of postictal EEG with and without IPA are shown in Figure 1A. IPA was noted following seizure termination in 26 seizures (84\%) in 15 patients (94\%), including six of the seven seizures with asynchronous electrographic offsets, with a mean duration of $36.2 \pm 21.4$ seconds (range 2-100 seconds, Table 1). An illustrative example is shown in Figure 1B. Figure 1C shows one of the five seizures (16\%) without IPA, demonstrating both ongoing background activity following seizure termination as well as superimposed focal low amplitude spike discharges. IPA was less common in Type 2 seizures compared to Type 1 and 3 seizures (Fisher's exact test, $\mathrm{N}=4,31, \mathrm{p}=0.007$ ). IPA duration was greater in Type 1 seizures compared to Type 2 or 3 seizures (Wilcoxon rank sum test, $\mathrm{N}=11,31, \mathrm{p}=0.006$ ). 
By our definition of IPA, attenuation had to be present in all recording channels. However, in two seizures (Patient 13, Seizure b, and Patient 16), focal attenuation was present in the majority of channels $(43 / 49,88 \%)$ in one case and fewer than half of the channels (39/93, $42 \%$ ) in the other case. In one patient, the ongoing activity was limited to the mesial subtemporal sites, and in the other patient, the active area was more broadly distributed over lateral and basal temporal, parietal and occipital regions.

In 19 of the 26 seizures with IPA (73\%), the IPA pattern was followed by a second postictal phase, characterized by ongoing attenuation interrupted at intervals by mixed-frequency, synchronized or asynchronous bursting (Figure 2). This burst-attenuation phase began abruptly, and typically simultaneously at all recorded channels. Bursts were composed of theta and delta frequency activity of varying amplitudes, and at times included focal or widespread epileptiform discharges confined to the bursts. This pattern transitioned to the third phase, in which a continuous background resumed, i.e. the periods of attenuation were no longer seen. In the seven seizures lacking the burst-attenuation stage, IPA transitioned directly to a continuous background (Figure 1B). The total time from seizure termination to resumption of a continuous background was $72.2 \pm 43.2$ seconds (range 8-176 seconds).

We used power spectral analysis to investigate the frequency-specific shifts during IPA (Figure 3). As expected, there was a reduction in power compared to the pre-ictal baseline in frequencies below gamma range during IPA. However, in the gamma band, power was increased during IPA in 22 of the 25 seizures with adequate preictal data for determining baseline (Figure 3A-B). Notably, no extracerebral source of the gamma activity including patient movements or staff interventions could be identified on video review (Figure 2). The gamma activity appeared time-invariant, persisting for the entire duration of IPA with little or no appreciable amplitude variability, and ceased abruptly at the end of IPA (Figure 1A-B, Figure 3A). Close inspection also revealed differences in waveform morphology and timing between channels (Figure 1B, left black arrow). In some cases, gamma power varied markedly across channels (Figure 3C-D). On average, 59+/-42 recording channels per seizure demonstrated increased IPA high frequency power compared to the pre-ictal baseline, with a significant overall increase in 22 of the 25 seizures (range 2-85 channels per seizure, paired Wilcoxon rank-sum test, all $\mathrm{p}<0.002$, i.e. $\mathrm{p}<0.05$ with Bonferroni correction). However, the distribution was skewed toward the extremes. Postictal gamma power increases were seen in more than $90 \%$ of channels in 13 seizures (52\%), and in fewer than $10 \%$ of channels in three seizures $(12 \%)$. The remaining nine seizures $(36 \%)$ showed mixed results, with 24-79\% of channels demonstrating increased postictal gamma power compared to the preictal baseline (Table 1). With the transition to the burst-attenuation phase, there was intermittent reappearance of the gamma activity, during which ongoing lower frequency activity, such as focal spiking, was seen to cease temporarily (Figure 2).

\section{Postictal clinical features}

The relationships between postictal EEG transitions and clinical features are depicted in Figure 4 . The predominant post-ictal clinical feature in most cases (27 seizures, $87 \%$ ) was immobility. We were able to distinguish two types of immobility: rigid and flaccid. Rigid immobility was characterized by rigid body or limb posture persisting despite staff 
interventions e.g. moving a limb. Rigid immobility was also associated with transient episodes of dystonic posturing or isolated myoclonic jerking. In flaccid immobility, the patient appeared limp with normal or reduced muscle tone noted due to staff interventions, with no spontaneous movements detected.

Flaccid immobility was seen in $18(58 \%)$ seizures in $12(75 \%)$ patients, including three seizures without IPA (Figure 4). This feature always began immediately after seizure termination, with an average duration of 38.2+/-23.3 seconds (range 4-90 seconds). In the seizures with IPA, flaccid immobility ended on average $12.1 \pm 27.5$ seconds after the end of IPA. Flaccid immobility ended up to 45 seconds before the end of IPA and as long as 49 seconds after IPA, ending prior to the end of IPA in four seizures. Movements signifying the termination of flaccid immobility consisted of myoclonic jerking, dystonia, or purposeful movements.

Rigid immobility was seen in 9 seizures (29\%) in $6(38 \%)$ patients, all of whom also had IPA. Rigid immobility had a similar temporal association with IPA as did flaccid immobility. It began immediately after seizure termination and had average duration of $47.6 \pm 37.6$ seconds (range 2 to 128 seconds). Rigid immobility ceased as early as 32 seconds before the end of IPA and as late as 28 seconds after IPA, ending prior to the end of IPA in three of the 9 seizures. There was no significant difference between flaccid and rigid immobility end times relative to the IPA-to-bursting transition (Wilcoxon rank sum test, $\mathrm{p}=0.23$ ).

Automatisms were seen postictally in seven seizures, including oral or manual automatisms, unilateral or bilateral blinking, shrugging, and repetitive bilateral leg flexion/bicycling. In two seizures (Patient 8, Seizure b and Patient 16), automatisms immediately followed flaccid immobility, and in two others (Patient 4, Seizure a, and Patient 5) they immediately followed rigid immobility. In three of the four seizures in which automatisms followed immobility, they occurred during the burst-attenuation phase. In two seizures (Patient 9, Seizure a, and Patient 12), automatism onset was delayed, occurring after the return to a continuous EEG background. In the last case (Patient 14, Seizure b), right hand automatisms and left hand dystonia began 24 seconds prior to electrographic seizure termination and continued until 5 minutes and 18 seconds after electrographic offset, accompanied by right eye deviation. One of the authors (CAS) was present in the patient room and interpreted the event clinically as an intracranial EEG-negative focal seizure. This was a nonlesional case with broad right frontal and temporal subdural electrode coverage. Following right frontal resection, the outcome was classified as Engel 1D (rare bilateral tonic-clonic seizures on anticonvulsant medication withdrawal) at 8 years postoperatively. Other postictal semiologies noted included repetitive (6 seizures) or isolated (11 seizures) jerking, and head and eye deviation with eye opening ( 3 seizures).

\section{Discussion}

This retrospective video-EEG study of intracranially-recorded FBTCS revealed a complex mixture of electroclinical activity in the postictal period. Rather than a simple presence or absence of ictal EEG activity, there was postictal pattern evolution characterized by abrupt electrographic transitions, and a variety of clinical motor manifestations, which were often 
dissociated from the striking EEG features. Electrographically, the postictal period was characterized by two distinct phases: IPA, with a persistent, invariant pattern of reduced power at the low end of the frequency range and often increased gamma power, followed in some cases by postictal bursting, in which the IPA pattern persisted but was interrupted by bursts of mixed frequency, predominantly slow activity, prior to return to a continuous background. Our findings indicate that marked low frequency attenuation following seizure termination, which may be a contributor to scalp EEG observations of postictal suppression, is common in FBTCS and appears to be a stage in a predictable sequence during electrographic postictal recovery.

IPA proved to be a nearly ubiquitous finding in our data set. IPA was detected in the majority of seizures (84\%) and in nearly all patients (94\%) in our study. Focal IPA, or an IPA-like pattern restricted to a portion of the recording area, was noted in an additional two seizures. The only patient in whom IPA or focal IPA was not observed (Patient 16) had only one seizure recorded. This is consistent with a previous study reporting that the occurrence of PGES in scalp EEG recordings is dependent on the number of FBTCS seizures recorded ${ }^{16}$. We also found that IPA occurrence and duration may be associated with semiology patterns that define BTCS type, suggesting common or linked mechanisms. Taken together, these data suggest that postictal attenuation, and the mechanisms that produce it, are typical features of FBTCS, rather than being exceptional findings. If true, this would suggest that postictal EEG attenuation contributes little to risk stratification for rare adverse outcomes, such as SUDEP.

We found no evidence in our study of slowly spreading depression, which has been detected following seizure termination in rodent models. ${ }^{17}$ Rather, the transitions between ictal, attenuation, and bursting states were abrupt and simultaneous across large brain regions in all cases. We hypothesized that widespread, simultaneous alteration of the frequency-power curve during IPA (Figure 3B) reflects projected activity from a remote, unmonitored site, as significant localized membrane current shifts occurring diffusely and simultaneously seems unlikely.

The presence of prominent, widespread gamma oscillations during IPA initially appeared to be artefactual as is commonly assumed. On closer examination, several observations favored a cerebral (if unknown) source. First, differences in waveform morphology and timing between channels were noted, especially at the onset of the gamma activity (Figure 1A-B). Second, patient movements did not correlate with the increased high frequency activity (Figure 2), and it was seen irrespective of the type of activity observed (e.g. flaccid immobility vs. dystonic posturing or myoclonic jerks). Third, the amplitude of the high frequency activity was remarkably consistent throughout the postictal attenuation period (Figure 1A). In contrast, myogenic artifact would be expected to show considerable temporal amplitude variation, ${ }^{18}$ and the same is typically true of environmentally induced artifacts. Fourth, we observed a contextual temporal pattern of continuous, invariant activity for the duration of IPA followed by intermittent reappearance, during which ongoing activity, such as focal spiking, was seen to cease temporarily (Figure 2). 
Postictal immobility, presumed to reflect depressed arousal and/or motor function ${ }^{19,20}$, is commonly observed after FBTCS and has been associated with postictal EEG attenuation in scalp recordings. Interestingly, the timing of postictal immobility with respect to IPA was highly variable. We observed immobility to appear as either a negative (flaccid) or positive (rigid) phenomenon. Flaccid immobility was more common (58\% of seizures) than rigid immobility (29\% of seizures), but both had similar temporal characteristics with respect to IPA. However, the two patterns of postictal immobility may have different implications for postictal brain activity. While flaccid immobility is potentially consistent with assumptions of reduced postictal neuronal activity, rigid immobility with associated dystonic posturing raises the possibility of an ongoing active process driven from an unmonitored source in either cortical or subcortical structures. Further, the fact that immobility may resolve during, or may outlast IPA suggests that they are driven by different but related processes.

A surprising result was the frequent observation of clinical seizure semiologies continuing or emerging after seizure termination. In aggregate, clinical manifestations including dystonic posturing, automatisms, head and eye deviation, and myoclonic jerking were observed either during IPA (13 seizures, $42 \%$ ), during the bursting phase (15 seizures, $48 \%$ ), or after return to continuous background but within the first minute after seizure termination (10 seizures, $32 \%$ ). Additionally, the presence of automatisms during the bursting or continuous phases, suggests that they are part of the progression of the ictal event. Persistent or reemerging automatisms have been observed within the first few seconds following focal onset seizures $^{21,22}$, but otherwise are widely considered to be specific for seizure activity. We speculate that these phenomena are evoked directly or indirectly by ongoing EEG-negative activity following apparent seizure termination, including during the IPA pattern and during immobility of either type.

Taking the electrographic and clinical data together with existing literature, we further speculate that IPA and its associated clinical semiologies are driven from subcortical, possibly brainstem, structures. A source located in areas that are commonly sampled with scalp or intracranial electrodes, e.g. cortical structures, remains possible but is difficult to reconcile with existing characterizations of postictal electroclinical events. Subcortical structures have been implicated as having a causative role in ictal loss of awareness ${ }^{23}$ and complex motor manifestations. ${ }^{24}$ Subcortical, particularly brainstem, activity has also been linked with cortical gamma oscillations. A recent study of intracranially implanted patients demonstrated coherence between respirations and the EEG gamma envelope recorded from limbic gray matter structures such as hippocampus and insula. ${ }^{25}$ Increased cortical gamma activity was seen with selective stimulation of the pedunculopontine tegmental nucleus in a rat model of acute hippocampal seizures. ${ }^{26}$ In a 1958 study by Gastaut of convulsive seizures in an in vivo cat strychnine/anoxia model, a pattern of cortical and hippocampal attenuation with superimposed high frequency activity in bilateral cortex but not in hippocampus, was seen concurrently with ongoing ictal discharges recorded from the bulbar reticular formation. ${ }^{27}$ Ictal brainstem recordings have also been reported in an in vivo rodent 4-aminopyridine model. ${ }^{28}$ Together, these results suggest that brainstem activity can drive both cortical EEG patterns and seizure semiologies - a view that is opposite to the more common interpretation of diffuse postictal EEG attenuation as indicative of globally reduced neuronal activity. ${ }^{3}$ Further investigation is required to define the mechanisms of prominent 
postictal semiologies such as rigid and flaccid immobility, and of IPA's unique combination of increased gamma activity and attenuation of sub-gamma frequencies.

\section{Limitations}

Our study, while taking advantage of the better signal to noise ratio of intracranial recordings, is limited by lack of simultaneous broad scalp coverage. Thus, we cannot directly compare IPA with the better known PGES phenomenon other than statistically. A prior report demonstrated that PGES identified from scalp recordings commonly co-exists with ongoing ictal or interictal activity in a simultaneous intracranial recording. ${ }^{29}$ The blind spots of scalp EEG are well known, and include electrographic activity in some areas difficult to access with extracranial sensors (subfrontal, subtemporal, orbitofrontal) or activity that is limited to small regions. ${ }^{12,29}$ Further, our study indicates that IPA may be spatially restricted in some cases. These factors likely contribute to the limited inter-reader reliability of PGES. ${ }^{30} \mathrm{~A}$ combined approach may be preferred for future studies.

It is notable that four seizures (13\%) in this series were provoked by cortical electrical stimulation mapping. As this is not typically reported in studies of postictal phenomena, it is uncertain whether this proportion is unusual. We note, however, that all but one of the patients (Patient 16) with provoked seizures also had unprovoked seizures that met study criteria.

\section{Conclusions}

Our study has implications for seizure propagation pathways and distant projections to or from subcortical structures mediating post-seizure electroclinical features, and raises important questions about the mechanisms of electrophysiology patterns after seizure termination.

\section{Acknowledgments}

This study was supported by NIH/NINDS (R01 NS084142 to CAS), and Citizens United for Research in Epilepsy (CURE) Henry Lapham Memorial Award (to LMB, CAS).

Disclosure of Conflicts of Interest

C. Bazil has received honoraria for consulting from UCB Pharma, Purdue Pharma and Aquestive. A. Pack has received research support from NIH (U01-NS038455), and receives royalties from Up to Date. H. Choi has received research support for investigator-initiated studies from Eisai, Sunovion and Acorda Therapeutics. S. Srinivasan receives clinical trial funding from Biogen and Neuropace. G. McKhann has research support from CURE (Prevention of Acquired Epilepsies Award) and NIH (R01-CD014279, R01-NS089552, and R01-NS084142). C. Schevon has research support from NIH (R01-NS084142) and CURE (Henry Lapham Memorial Award, with L. Bateman). L. Bateman has research support from NIH (U01-NS090407) and CURE (Henry Lapham Memorial Award, with C. Schevon). The remaining authors have no conflicts of interest to disclose.

\section{References}

1. Afra P, Jouny CC, Bergey GK. Termination patterns of complex partial seizures: An intracranial EEG study. Seizure. 2015; 32:9-15. [PubMed: 26552555]

2. Kaibara M, Blume WT. The postictal electroencephalogram. Electroencephalogr Clin Neurophysiol. 1988; 70(2):99-104. [PubMed: 2456198] 
3. Lhatoo SD, Faulkner HJ, Dembny K, et al. An electroclinical case-control study of sudden unexpected death in epilepsy. Ann Neurol. 2010; 68(6):787-96. [PubMed: 20882604]

4. Ryvlin P, Nashef L, Lhatoo SD, et al. Incidence and mechanisms of cardiorespiratory arrests in epilepsy monitoring units (MORTEMUS): a retrospective study. Lancet Neurol. 2013; 12(10):96677. [PubMed: 24012372]

5. Wei Y, Ullah G, Schiff SJ. Unification of neuronal spikes, seizures, and spreading depression. J Neurosci. 2014; 34(35):11733-43. [PubMed: 25164668]

6. Pacia SV, Ebersole JS. Intracranial EEG Substrates of Scalp Ictal Patterns from Temporal Lobe Foci. Epilepsia. 1997; 38(6):642-54. [PubMed: 9186246]

7. Tao JX, Baldwin M, Ray A, et al. The impact of cerebral source area and synchrony on recording scalp electroencephalography ictal patterns. Epilepsia. 2007; 48(11):2167-76. [PubMed: 17662060]

8. Ray S, Maunsell JHR. Different origins of gamma rhythm and high-gamma activity in macaque visual cortex. PLoS Biol. 2011; 9(4).

9. Weiss SA, Banks GP, McKhann GM, et al. Ictal high frequency oscillations distinguish two types of seizure territories in humans. Brain. 2013; 136(12):3796-808. [PubMed: 24176977]

10. Smith EH, Liou J-Y, Davis TS, et al. The ictal wavefront is the spatiotemporal source of discharges during spontaneous human seizures. Nat Commun. 2016; 7:11098. [PubMed: 27020798]

11. Penfield W, Jasper H. Epilepsy an the Functional Anatomy of the Human Brain. Boston: Little, Brown; 1954.

12. Tao JX, Ray A, Hawes-Ebersole S, et al. Intracranial EEG substrates of scalp EEG interictal spikes. Epilepsia. 2005; 46(5):669-76. [PubMed: 15857432]

13. Alexandre V, Mercedes B, Valton L, et al. Risk factors of postictal generalized EEG suppression in generalized convulsive seizures. Neurology. 2015; 85(18):1598-603. [PubMed: 26333799]

14. Semmelroch M, Elwes RDC, Lozsadi DA, et al. Retrospective audit of postictal generalized EEG suppression in telemetry. Epilepsia. 2012; 53(2).

15. Weiss SA, Lemesiou A, Connors R, et al. Seizure localization using ictal phase-locked high gamma: A retrospective surgical outcome study. Neurology. 2015; 84(23):2320-8. [PubMed: 25972493]

16. Lamberts RJ, Gaitatzis A, Sander JW, et al. Postictal generalized EEG suppression: An inconsistent finding in people with multiple seizures. Neurology. 2013; 81(14):1252-6. [PubMed: 23966251]

17. Aiba I, Noebels JL. Spreading depolarization in the brainstem mediates sudden cardiorespiratory arrest in mouse SUDEP models. Sci Transl Med. 2015; 7:282ra46.

18. Andrade-Valenca LP, Dubeau F, Mari F, et al. Interictal scalp fast oscillations as a marker of the seizure onset zone. Neurology. 2011; 77(6):524-31. [PubMed: 21753167]

19. Semmelroch M, Elwes RDC, Lozsadi DA, et al. Retrospective audit of postictal generalized EEG suppression in telemetry. Epilepsia. 2012; 53(2):e21-4. [PubMed: 22050242]

20. Kuo J, Zhao W, Li CS, et al. Postictal immobility and generalized EEG suppression are associated with the severity of respiratory dysfunction. Epilepsia. 2016; 57(3):412-7. [PubMed: 26763069]

21. Rásonyi G, Fogarasi A, Kelemen A, et al. Lateralizing value of postictal automatisms in temporal lobe epilepsy. Epilepsy Res. 2006; 70(2):239-43. [PubMed: 16765567]

22. Devinsky O, Kelley K, Yacubian EMT, et al. Postictal Behavior: A clinical and subdural electroencephalographic study. Arch Neurol. 1994; 51(3):254-9. [PubMed: 8129636]

23. Motelow JE, Li W, Zhan Q, et al. Decreased subcortical cholinergic arousal in focal seizures. Neuron. 2015; 85(3):561-72. [PubMed: 25654258]

24. Miller JW. The role of mesencephalic and thalamic arousal systems in experimental seizures. Prog Neurobiol. 1992; 39(2):155-78. [PubMed: 1502335]

25. Herrero JL, Khuvis S, Yeagle E, et al. Breathing above the brainstem: Volitional control and attentional modulation in humans. J Neurophysiol. 2017; 119(1):145-59. [PubMed: 28954895]

26. Furman M, Zhan Q, McCafferty C, et al. Optogenetic stimulation of cholinergic brainstem neurons during focal limbic seizures: Effects on cortical physiology. Epilepsia. 2015; 56(12):e198-202. [PubMed: 26530287]

27. Gastaut H, Naquet R, Fischer-Williams M. The pathophysiology of grand mal seizures generalized from the start. J Nerv Ment Dis. 1958; 127(1):21-33. [PubMed: 13564242] 
28. Salam MT, Montandon G, Genov R, et al. Mortality with brainstem seizures from focal 4aminopyridine-induced recurrent hippocampal seizures. Epilepsia. 2017; 58(9):1637-44. [PubMed: 28691204]

29. Altenmüller DM, Schulze-Bonhage A, Elger CE, et al. Local brain activity persists during apparently generalized postictal EEG suppression. Epilepsy Behav. 2016; 62:218-24. [PubMed: 27494359]

30. Theeranaew W, McDonald J, Zonjy B, et al. Automated detection of postictal generalized EEG suppression. IEEE Trans Biomed Eng. 2018;. 


\section{Key Points}

1. IPA, whether diffuse or focal, should be regarded as an ubiquitous feature of tonic-clonic seizures, rather than an unusual event.

2. Two types of immobility, flaccid and rigid, may be seen following FBTCS but do not precisely correlate with IPA.

3. Clinical seizure semiologies, such as automatisms, may continue or newly emerge following FBTCS.

4. Gamma activity coexisting with IPA suggests ongoing neuronal activity outside recorded areas, possibly in subcortical structures. 
A
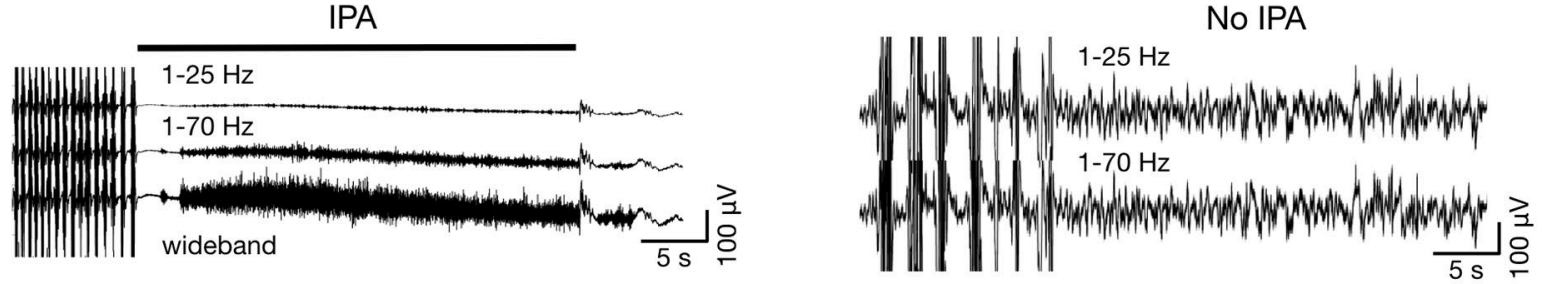

B
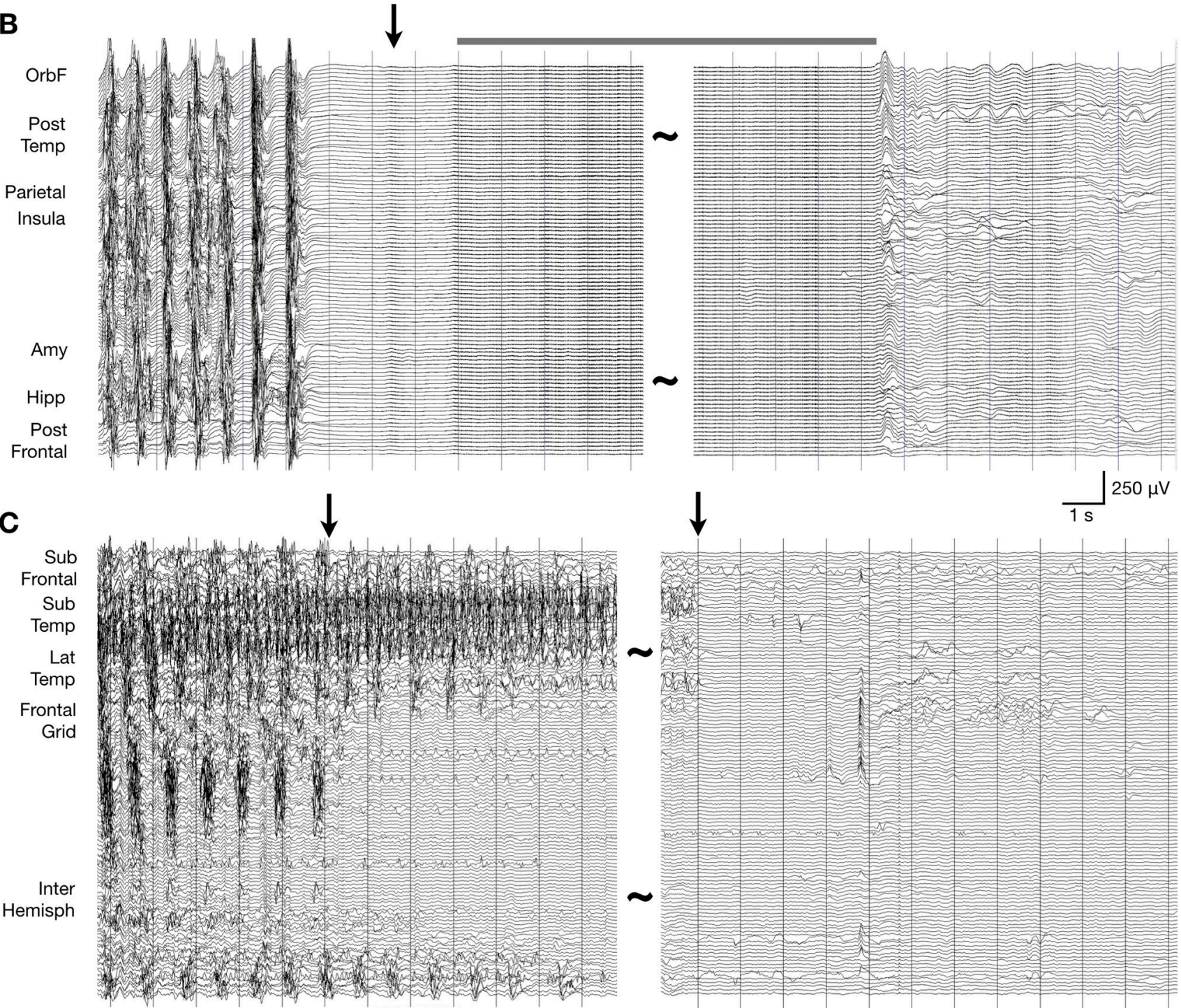

Figure 1: Characteristics of intracranial postictal attenuation (IPA).

A) Single channel examples of postictal recordings with (left) and without (right) IPA, with different filter settings (FIR order 90) as shown. The $1-70 \mathrm{~Hz}$ filter replicates the trace depicted in EEG review software at typical user-defined settings. In the IPA channel, there is no discernable activity in the $1-25 \mathrm{~Hz}$ filtered signal. The activity seen in the other two traces is in the gamma range, and remains below the $50 \mu \mathrm{V}$ threshold in the $1-70 \mathrm{~Hz}$ trace. Note that the gamma activity ceases after the transition to continuous slow activity. In contrast, delta and theta activity is prominent in the non-IPA channel, and there is relatively 
little gamma activity. B) IPA in a seizure from Patient 9, with low-frequency filter $0.5 \mathrm{~Hz}$ and high frequency filter off. Vertical lines delineate seconds. For ease of illustration, sensitivity was set to $100 \mu \mathrm{V} / \mathrm{mm}$, and only the first and last few seconds of the 34-second IPA period are shown. During IPA, the superimposed gamma-range activity is visible, with an initial brief burst (black arrow) followed a second later by sustained, amplitude-invariant activity (gray bar). Small variable inter-channel latencies can be seen during the initial gamma burst. IPA then ceases abruptly and simultaneously across all channels, accompanied by reduction in superimposed gamma activity. C) Seizure from patient 14 showing asynchronous termination (black arrows; first offset in lateral frontal contacts, second offset in subtemporal, lateral temporal, and subfrontal contacts). After final electrographic termination, there was no evidence of either IPA or increased postictal gamma activity. 


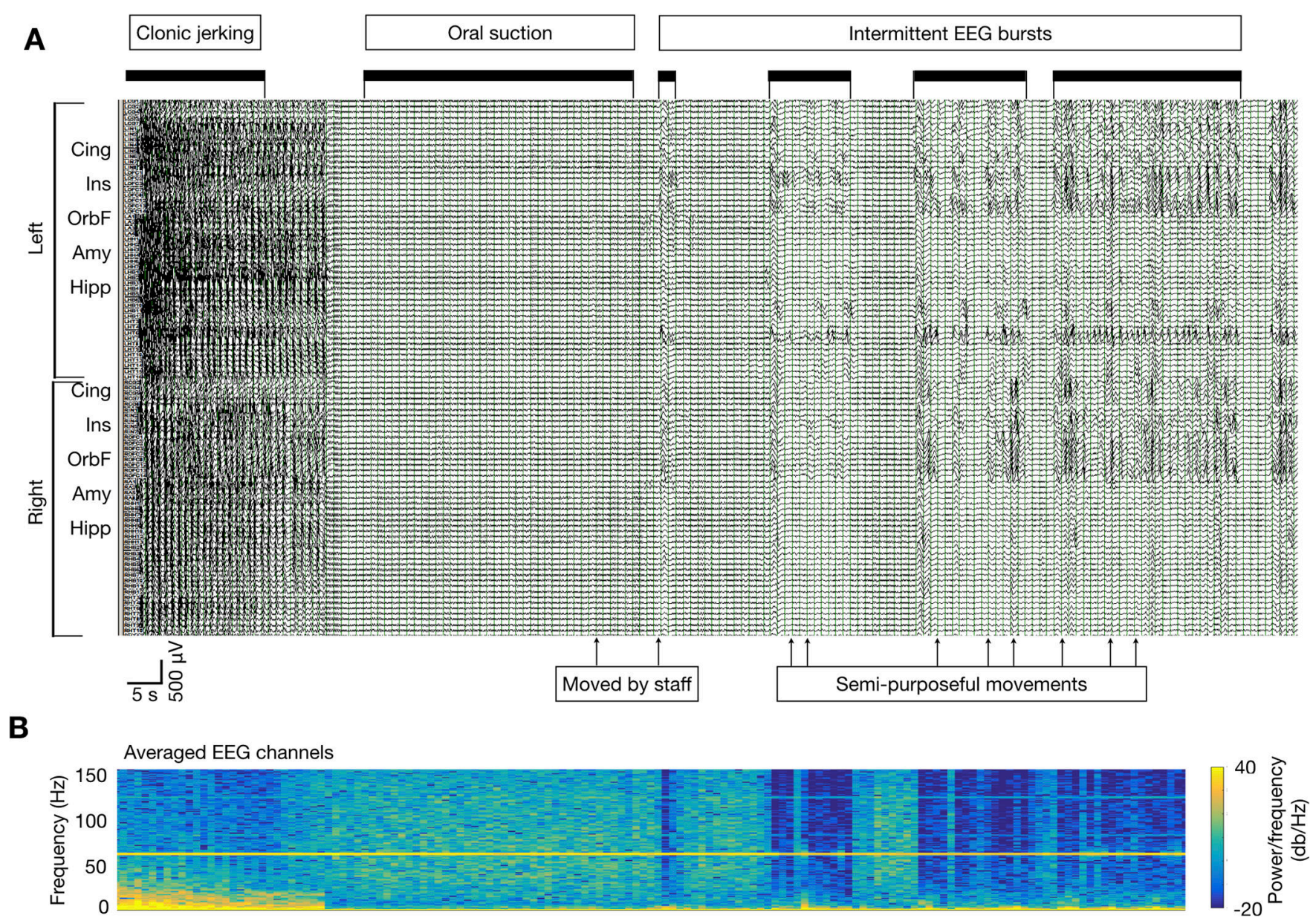

Figure 2: Discontinuous bursting following IPA with clinical correlation.

A) Seizure from patient 8 with IPA and superimposed gamma activity, shown in temporally compressed view to present all postictal stages. Filter settings are as in Figure 1. Sensitivity was set to $150 \mu \mathrm{V} / \mathrm{mm}$ for clarity of presentation. After termination of the continuous IPA pattern, there are bursts of diffuse slowing in which superimposed persistent gamma activity is no longer seen, but periodic delta waves and sharp wave discharges are present (labeled black bars). This background is disrupted by periodic, brief re-emergence of the previous IPA pattern, during which the superimposed gamma transiently reappears, and ongoing paroxysmal activity ceases. The timing of observed movements or interventions (clonic jerking, suctioning, patient moved by staff) are marked. Spontaneous, semi-purposeful patient movements were noted occasionally during periods of diffuse slowing, but not during IPA/gamma activity re-emergence. The EEG activity, including terminal ictal discharges and the IPA pattern with superimposed high frequencies, does not appear to be affected by movements and interventions noted on video. B) Time-frequency spectrogram $(1-150 \mathrm{~Hz})$ of averaged EEG data across all channels, temporally aligned with the EEG shown in A. 
A
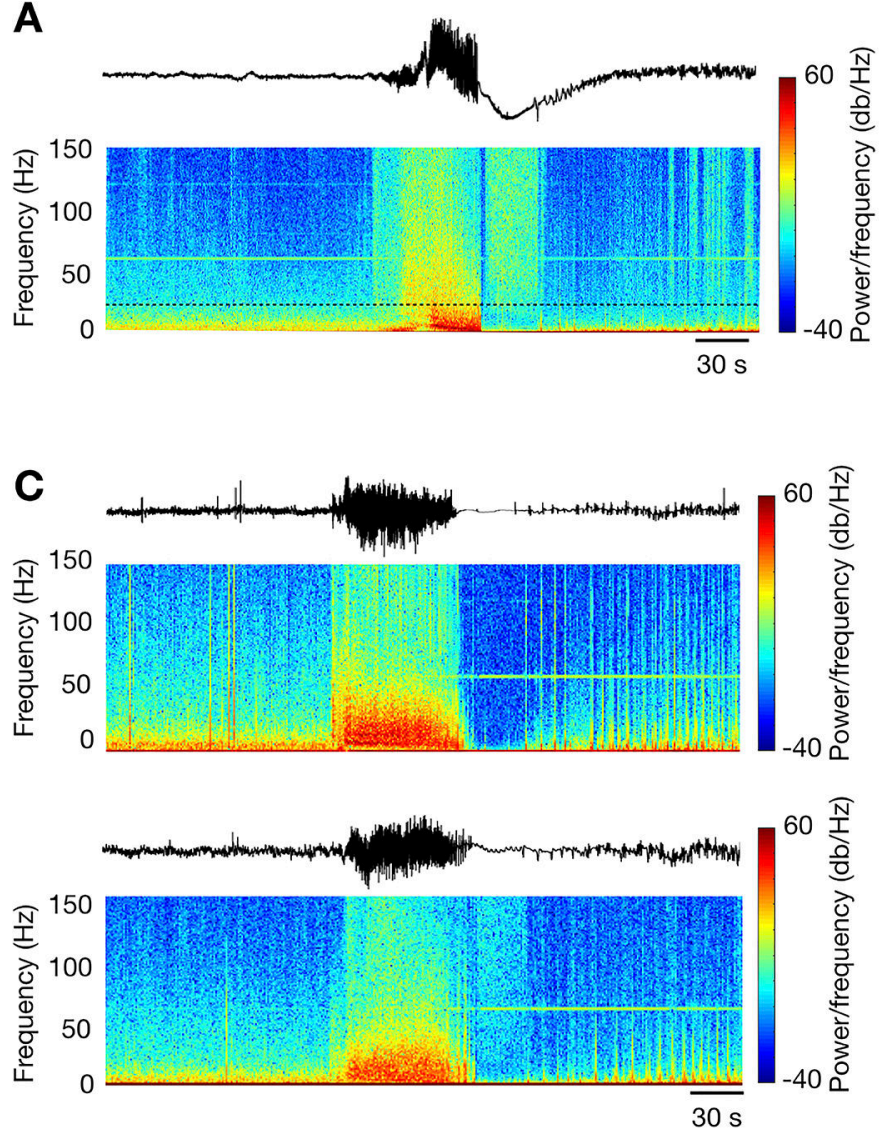

B
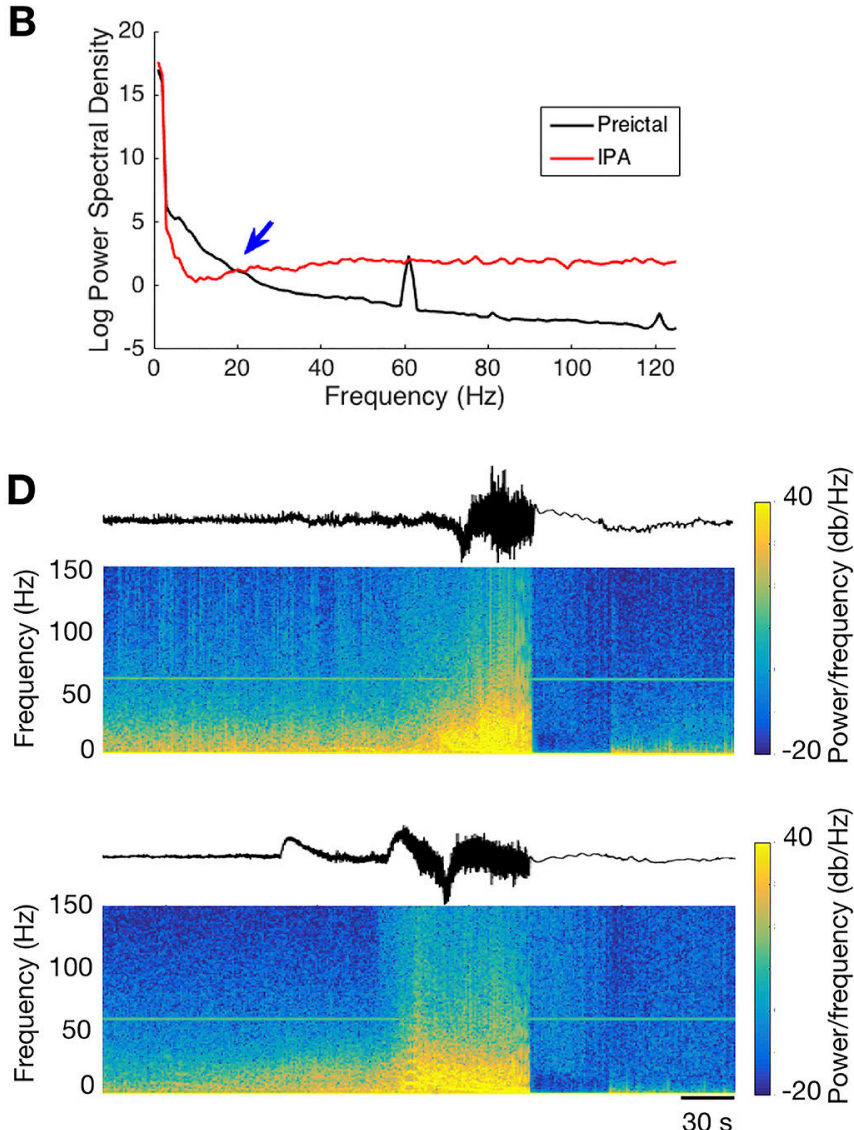

Figure 3: Spectral characteristics of IPA-associated gamma activity.

A) Time-frequency spectrogram of a peri-ictal recording aligned with the EEG trace for a sample channel from Patient 9, demonstrating the expected broadband power increase during the seizure. During IPA, there is a marked increase in power above the "Berger band" range of $1-25 \mathrm{~Hz}$ despite the reduced power in lower frequencies (below the black dashed line) that is the defining feature of IPA. B) Power spectral density from this same channel during preictal and IPA time periods, demonstrating the reduction in power below $25 \mathrm{~Hz}$, transitioning (blue arrow) to increased power in higher frequencies. C,D) Paired EEG channels recorded from the same seizure (Patient 4 in panel C, Patient 11 in panel D) with corresponding spectrograms, demonstrating marked cross-site differences in postictal gamma activity. In both cases, the top panel demonstrates postictal reduction in gamma activity compared to the preictal baseline, while the bottom panels demonstrate increased activity. This mixed pattern, with high frequency activity limited to $24-79 \%$ of channels, was present in nine of the 26 seizures (35\%) demonstrating IPA. 


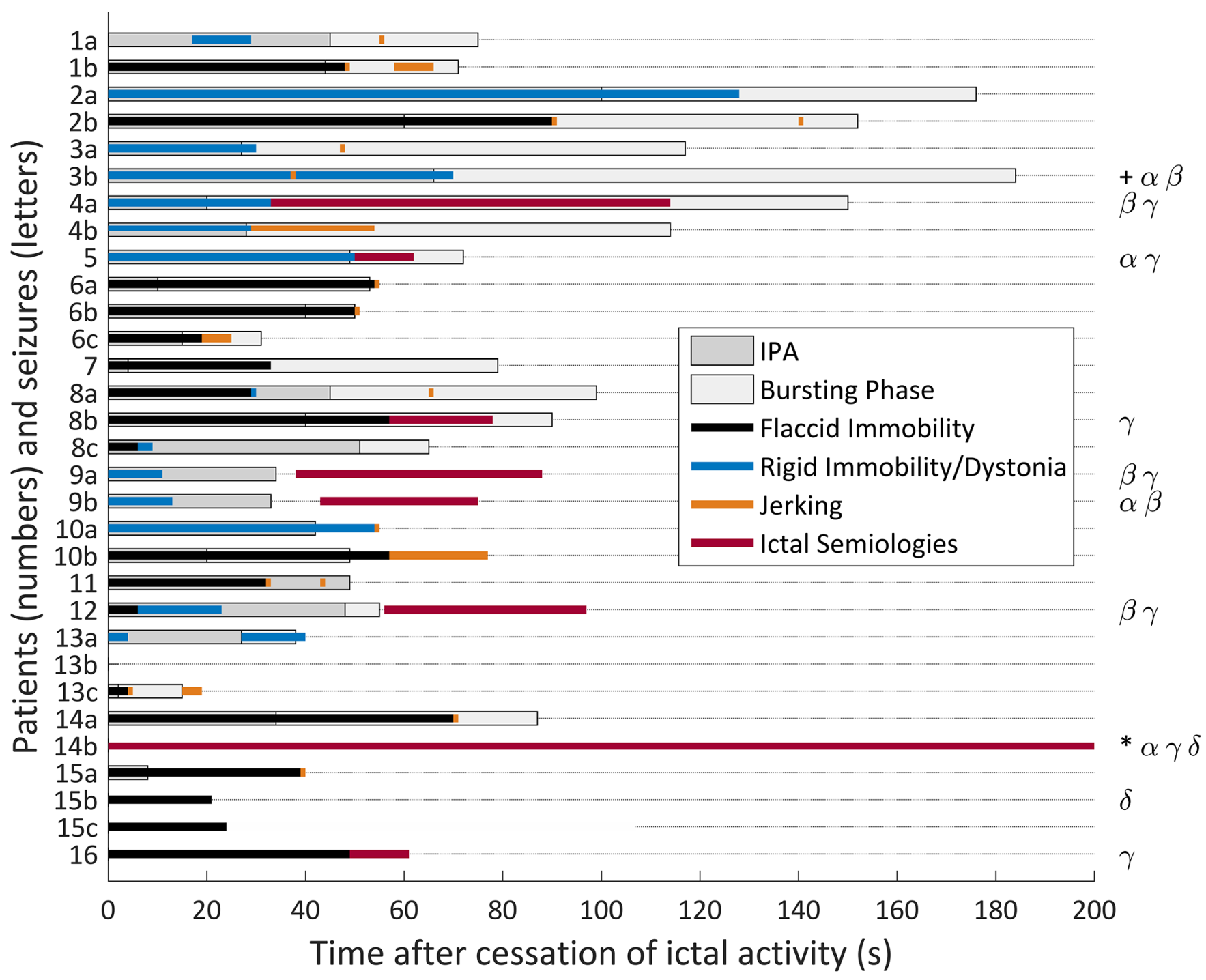

Figure 4. Clinical and electrographic features in the postictal period.

Bar chart showing time durations of IPA (dark gray bars) and the bursting phase (light gray) prior to the return to continuous EEG background. The superimposed colored bars depict time periods for flaccid immobility (black), rigid immobility with dystonic posturing (blue), repetitive or paroxysmal myoclonic jerking (orange), and other ictal semiologies (pink). The specific semiologies observed for each seizure are coded on the right side of the figure: dystonic posturing (a), myoclonic jerking $(\beta)$, automatisms $(\gamma)$, and head/eye deviation $(\delta)$. In the seizure marked with a ' + ', dystonic posturing with repetitive jerking was seen beginning at the time of the orange tick mark. In the seizure marked with a '*', a mixture of activities was noted. Right hand automatisms and left hand posturing were seen beginning prior to electrographic seizure termination and continuing postictally. Right eye deviation began 67 seconds after seizure termination. 
Table 1:

\section{Patient information}

\begin{tabular}{|l|l|l|l|l|l|l|}
\hline Patient & Age/ Gender & Implant/\#electrodes & FBTCS type & SOZ & IPA (s) & Gamma \% channels \\
\hline 1 & $20 / \mathrm{M}$ & LFT/84 & 1,1 & LT & 44,45 & 100,100 \\
\hline 2 & $50 / \mathrm{M}$ & BFT/32 & 1,3 & RF(1) RFT(1) & 100,60 & 100,100 \\
\hline 3 & $19 / \mathrm{F}$ & RH/126 & $3,1^{b}$ & RO & $27,66^{b}$ & $52,8^{b}$ \\
\hline 4 & $30 / \mathrm{F}$ & LH/121 & 3,3 & LFT & 20,28 & 68,24 \\
\hline 5 & $25 / \mathrm{M}$ & LFT/56 & 3 & LT & 49 & 79 \\
\hline 6 & $22 / \mathrm{M}$ & RFTP/102 & $3^{b}, 3,3^{b}$ & RF & $10^{b}, 40,15^{b}$ & $3^{b}, 59,2^{b}$ \\
\hline 7 & $20 / \mathrm{F}$ & LP/48 & 3 & LP & 4 & 57 \\
\hline 8 & $23 / \mathrm{M}$ & BFTC $/ 126$ & $3,1,1$ & RT(2) LT(1) & $45,40,51$ & $100,100,100$ \\
\hline 9 & $30 / \mathrm{F}$ & LFT $/ 110$ & 3,1 & LF & 34,33 & 100,100 \\
\hline 10 & $25 / \mathrm{M}$ & LT/RFT $/ 126$ & 3,3 & RT & 42,20 & 66,79 \\
\hline 11 & $37 / \mathrm{M}$ & RTPO/78 & 1 & RT & 49 & 69 \\
\hline 12 & $32 / \mathrm{F}$ & LFT/84 & $1 b$ & LT & $48^{b}$ & 93 \\
\hline $13 \mathrm{a}$ & $34 / \mathrm{M}$ & LT/50 & 1,2 & LT & $27,0^{d}$ & 93, no IPA \\
\hline $13 \mathrm{~b}$ & $35 / \mathrm{M}$ & LT/72 & 2 & LT & 2 & 100 \\
\hline 14 & $37 / \mathrm{M}$ & RFT/128 & $1,2^{b}$ & RF & $34,0^{b}$ & 98, no IPA $b$ \\
\hline 15 & $16 / \mathrm{M}$ & RH/70 & $3,3,3$ & RT & $8,0,0$ & $c$ no IPA, no IPA \\
\hline 16 & $22 / \mathrm{F}$ & LH/110 & 2 & LT & $0{ }^{d}$ & no IPA \\
\hline
\end{tabular}

FBTCS=focal to bilateral tonic-clonic seizure, $\mathrm{SOZ}=$ seizure onset zone, IPA=intracranial postictal attenuation, $\mathrm{L}=\mathrm{left}, \mathrm{R}=\mathrm{right}, \mathrm{B}=\mathrm{bilateral}$, $\mathrm{F}=$ frontal, $\mathrm{T}=$ temporal, $\mathrm{C}=$ central, $\mathrm{P}=$ parietal, $\mathrm{O}=$ occipital, $\mathrm{H}=$ hemispheric

$\stackrel{a}{=}$ stereo EEG implant

$b$ =provoked seizure

${ }^{c}=$ excluded from analysis due to preictal baseline EEG $<5$ minutes

${ }^{d}=$ partial postictal attenuation 